IOS Press

\title{
Editorial
}

\section{Geospatial semantics and linked spatiotemporal data - Past, present, and future}

\author{
Krzysztof Janowicz ${ }^{\mathrm{a}}$, Simon Scheider ${ }^{\mathrm{a}}$, Todd Pehle ${ }^{\mathrm{b}}$ and Glen Hart ${ }^{\mathrm{c}}$ \\ ${ }^{a}$ University of California, Santa Barbara, USA \\ ${ }^{\mathrm{b}}$ Orbis Technologies, USA \\ ${ }^{\mathrm{c}}$ Ordnance Survey, UK
}

\begin{abstract}
The Geosciences and Geography are not just yet another application area for semantic technologies. The vast heterogeneity of the involved disciplines ranging from the natural sciences to the social sciences introduces new challenges in terms of interoperability. Moreover, the inherent spatial and temporal information components also require distinct semantic approaches. For these reasons, geospatial semantics, geo-ontologies, and semantic interoperability have been active research areas over the last 20 years. The geospatial semantics community has been among the early adopters of the Semantic Web, contributing methods, ontologies, use cases, and datasets. Today, geographic information is a crucial part of many central hubs on the Linked Data Web. In this editorial, we outline the research field of geospatial semantics, highlight major research directions and trends, and glance at future challenges. We hope that this text will be valuable for geoscientists interested in semantics research as well as knowledge engineers interested in spatiotemporal data.
\end{abstract}

\section{Introduction and motivation}

While the Web has changed with the advent of the Social Web from mostly authoritative content towards increasing amounts of user generated information, it is essentially still about linked documents. These documents provide structure and context for the described data and easy their interpretation. In contrast, the evolving Data Web is about linking data, not documents. Such datasets are not bound to a specific document but can be easily combined and used outside of their original creation context. With a growth rate of millions of new facts encoded as RDF-triples per month, the Linked Data cloud allows users to answer complex queries spanning multiple, heterogeneous data sources from different scientific domains. However, this uncoupling of data from its creation con- text makes the interpretation of data challenging. Thus, research on semantic interoperability and ontologies is crucial to ensure consistency and meaningful results. Space and time are fundamental ordering principles to structure such data and provide an implicit context for their interpretation. Hence, it is not surprising that many linked datasets either contain spatiotemporal identifiers themselves or link out to such datasets, making them central hubs of the Linked Data cloud.

Prominent examples include Geonames.org as well as the Linked Geo Data project, which provides a RDF serialization of Points Of Interest from Open Street Map [103]. Besides such Voluntary Geographic Information (VGI), governments and governmental agencies recently started to develop geo-ontologies and publish their data as Linked Spatiotemporal Data [54]. Examples include the US Geological Survey [107] and 
the UK Ordnance Survey [41]. Furthermore, myriad other Linked Data sources contain location-based references. For instance, a dataset from the digital humanities may link information about exhibits to places and their historic names [76]. Following outgoing links, scholars can explore these places and learn about events which took place there. This historic events dataset may in turn link to information about physical objects and actors that were involved in these events.

To query data across different data sources requires information about the intended meaning of the used terms. In the example above, the datasets may use the CIDOC conceptual reference model [28] as a common top-level ontology that defines terms such as event or participatesIn. On the domain level, researchers have proposed ontologies, e.g., for Geology [17], that enrich top-level ontologies such as DOLCE with domain specific facts. However for highly heterogeneous domains and interdisciplinary research, dealing with geospatial data as well as establishing and maintaining such toplevel and domain-level ontologies may turn out to be difficult or even impossible. Therefore, a major challenge of semantic research in the context of Linked Data lies in exploiting semantic heterogeneity, instead of resolving it [52].

Datasets and ontologies are just two components of the Geospatial Semantic Web [30]. The formal semantics defined for knowledge representation languages such as the Web Ontology Language (OWL) support reasoning services that can make implicit facts explicit, discover incompatibilities, improve retrieval beyond keyword search, and provide the framework for complex integrity constraint checking that reduces the risk of combining incompatible data and models. Finally, all of this would be of little use if not supported by semantics-driven user interfaces and novel interaction paradigms that support the exploration of data, models, and services.

In the following we outline the research field of geospatial semantics, sketch its major research directions so far, and highlight future challenges. We hope that this overview will be valuable for geoscientists interested in semantics research as well as knowledge engineers interested in the geosciences.

\section{Geospatial semantics in a nutshell}

Geospatial semantics is a research area combining Geographic Information Science (GIScience), spatial databases, cognitive science, Artificial Intelligence (AI), and the Semantic Web [65]. It addresses the meaning of digital referents at a geographic scale, such as places, locations, events, and geographic objects in digital maps, geodatabases, and earth models. Geospatial semantics uses a variety of methods ranging from top-down knowledge engineering and logical deduction to bottom-up data mining and induction. It integrates knowledge engineering with methods specific to GIScience, such as spatial reference systems and spatial reasoning. It also extends methods that originated in cognitive science such as semantic similarity and analogy reasoning, e.g., to enable semantics-based geographic information retrieval [53]. Often, geospatial semantics combines work on conceptual modeling and geo-ontologies with spatial statistics, e.g., to study land cover [4].

The semantic interpretations of geographic information can differ considerably, which frequently causes misunderstandings when using and combining data and services on the Web. A well studied example are Web services that provide sensor data, e.g., from weather stations. For instance, in order to simulate the spread of a toxic gas plume, two different services may be queried for wind direction measurements. Both services may be syntactically comparable in that they return a string called wind direction as output together with an integer ranging from $0-360^{\circ}$. Nevertheless, both services can have contradicting semantic interpretations of what the returned values refer to: wind blows to or wind blows from. Thus, sending both values to an evacuation simulation running on a Web Processing Service (WPS) will yield misleading results [88]. Other examples include different and evolving conceptualizations of land cover types in the context of the Kyoto protocol [35] as well as geographic feature types such as forests or Points Of Interest. Besides challenges arising from integrating heterogeneous data and combining services, data-model intercomparison plays another crucial role [82]. Finally, time and the resulting change is another challenge that has to be taken into account. Most concepts are not static but evolve over time or are even dynamically redefined. For the long term preservation and maintenance of data and ontologies this leads to research challenges such as how to handle semantic aging [97].

One can distinguish two major strands of scientific thought in geospatial semantics, by analogy with Kuhn's [69] distinction between modeling vs. encoding on the Semantic Web. One is concerned with the design task of semantic modeling. It addresses the problem how geographic information should be modeled in an information ontology, i.e., which relations 
and classes are useful in order to discover, capture and query the meaning of spatiotemporal and geographic phenomena. Examples include work on geospatial ontology engineering $[34,13,60]$ and the formalization of spatial reasoning [22]. Spatial relations allow querying and localizing complex geometrical objects, such as cities or buildings, relative to other referents, such as countries and roads [56]. It was recognized early that such queries need to deal with indeterminate boundaries of geographic objects [19]. This research strand goes back to a tradition of work on spatial representations and operations in Geographic Information Systems (GIS) [20] as well as on integrity constraints in spatial databases.

Another strand is concerned with the task of semantics-based search, integration, and interoperability of geo-referenced information, as discussed in the examples before. It addresses the problem of how geographic referents can be semantically linked to other kinds of information with related meaning. Due to the vast heterogeneity of geo-data and models spanning fields such as human and cognitive geography, ecology, economics, geology, climatology, oceanography, transportation research, and so forth, integration and sharing of georeferenced information requires methods to ensure semantic interoperability [43]. Additionally, geographic information frequently needs to be represented on different levels of abstraction, scale, and granularity [32], and can be inherently vague and uncertain [11]. This creates another source of interoperability problems. An important challenge of semantic linking is how geospatial referents, such as events and places, can be automatically discovered in data sources which are not linked or georeferenced. Recent examples for work on querying includes GeoSPARQL as a common query language for the Geospatial Semantic Web as well as triple stores that can effectively handle and index Linked Spatiotemporal Data [9]. Other work along these lines also addressed the role of semantic similarity for spatial scene queries $[83,71]$.

\section{Major research directions}

In the following, we give a brief introduction into some of the major research topics in geospatial semantics and related areas.

\section{Geo-ontology engineering}

Geographic information deals with a variety of phenomena on a certain range of spatial scales. Even though geographic referents are rooted in diverse domains, they share certain semantic characteristics and principles that can be exploited in common approaches towards designing geo-ontologies. For example, such ontologies should support access to phenomena on flexible resolution levels and scales [8]. They also have to deal with the various natures of spatial boundaries [102]. Examples for top-level geo-ontologies that incorporate the principle of spatial granularity include the work of Bittner et al. [13]. Usually, such foundation ontologies are extended by domain ontologies, e.g., the SWEET ontology for earth and environmental science [89].

However, in recent time, it has become apparent that geographic concepts are situated and contextdependent [15], that they can be described from different, equally valid, points of view [51], and that ontological commitments are arbitrary to a large extent [93]. This makes standard comprehensive approaches towards ontology engineering more likely to fail. Semantic engineering, however, may be slightly redefined, namely as a method of communicating possible interpretations of data terms by constraining them towards the intended ones [68], without prescribing ontological commitments. For example, so-called ontology design pattern have been proposed and implemented as modular, flexible, and reusable building blocks (or strategies) that support engineers and scholars in defining local, purpose-driven ontologies [37]. Another approach is based on grounding vague terms with possibly multiple meanings [12]. Additionally, one can also engineer ontologies in a layered fashion $[34,25]$. Such a layered approach can start with observation procedures on the bottom level and then provide deductive and inductive methods to arrive at more abstract but reproducible ontological categories [51].

\section{Semantic reference systems}

In its most basic definition, geographic information contains a spatial, a temporal, and a thematic component [20]. The usefulness of geographic information lies, to large extent, in the availability of reference systems for the precise semantic interpretation of these components. Spatial reference systems provide the formal vocabulary to calculate with precise locations, e.g., in the form of points on a mathematical ellipsoid, as well as with their meaning in terms of technical operations. The latter are given in terms of geodetic datums, i.e., standard directions and positions 
of the ellipsoid, which allow interpreting locations as results of repeatable measurements on the earth surface. Both is required to make sense out of spatial data. Temporal reference systems, such as calendars, similarly handle the representation of time, and allow to translate between different calendars. The thematic (also called attributive) component of geographic information requires reference systems as well [20]. In analogy, Kuhn proposed the generalized notion of Semantic Reference Systems (SRS) [64], which enable a precise interpretation of all components of geospatial data in terms of measurement scales and observation procedures. For example, attribute values such as the wind directions discussed before can be interpreted in terms of reference systems for cardinal wind directions and anemometers. Establishing such SRS, their standard operations as well as their formal vocabularies, is an ongoing research topic $[87,93,68]$, and has been named among the most important and challenging projects of GIScience [78].

\section{Semantic primitives and information grounding}

Related to SRS is the problem on which level of $a b$ straction a geospatial dataset can be semantically described in order to convey its meaning and to compare it with other datasets. As discussed above, geospatial ontologies reflect different world views on different levels of abstraction for good reasons. However, in order to compare and link them, one needs a common semantic plane. What are the basic concepts on which the primitive notions in a geospatial ontology should be founded? What are useful cognitive abstractions that can be reused across different ontologies? What are useful semantic backgrounds that enable comparison of different ontologies and conceptualizations with each other?

One approach towards geospatial semantic primitives is based on spatial cognitive schemas. For example, Johnson's [55] image schemas, such as container, or path, are cross-domain abstractions (i.e., conceptual metaphors) underlying many different kinds of geographic data such as road networks or administrative boundaries. Thus, they can be used for designing core concepts in geospatial ontologies [66]. Lynch's urban patterns [75] and Alexander's design patterns [6] may be seen from a similar angle. Likewise, Gärdenfors' notion of cognitive categories as convex regions in a conceptual space [38] is a cognitive schema that can be exploited for comparison of geospatial concepts $[90,2]$.
Another approach acknowledges that cognitive concepts are themselves abstractions and, thus, in need of grounding in the sense of Harnad [42]. Geographic information concepts may be grounded in terms of embodied perceptual routines, perception-action cycles, and situated simulations [7]. Perception-action cycles underlie Gibson's meaningful environment and his central notion of affordance [39]. Both can be used to understand geographic media, such as road networks, in terms of the kinds of actions they afford [95,96]. More generally, it is possible to understand the meaning of geographic information in a pragmatic sense [14], e.g., in terms of repeatable actions taken to generate a dataset [93], as well as in a teleological sense, i.e., in terms of the underlying purpose [25]. Relevant actions may involve cognitive constructions, which account for abstract notions, as well as perceptual operations, which allow humans to reliably simulate and predicate some phenomenon in jointly observable environmental scenes [93].

\section{Event discovery and spatiotemporal ontologies}

Geographic information is inherently temporal in the sense that geographic assertions, such as partonomic relations between administrative regions or the membership in organizations, are valid only over a certain period [59]. Consequently, research investigates how this temporal dimensions can be brought into geospatial data. This is especially crucial for the integration of Linked Data on the Web. To give a concrete example, problems arise when administrative regions are linked via OWL:sameAs, and their properties, such as population numbers, are not temporally indexed, e.g., via blank nodes.

Over the last years a multitude of work on spatiotemporal modeling, temporal GIS [21], and simple temporal gazetteer models [47] has been published. Research also addressed event ontology design patterns [110]. However, a particular challenge remains the automated detection of events from observation data on a geographic scale [10], such as rainstorms or blizzards [27]. Examples of work on geographic event detection and identification algorithms include the work by Agouris and Stefanidis [3]. Nonetheless, there remain open questions. For example, regarding general formal and computational procedures of geographic event detection, concerning the tight coupling of geospatial ontologies with detected events, as well as the triggering of data and ontology updates by automatically detected events [72]. These challenges 
are reflected, to some extent, in ontological questions about the relationship between processes, objects, and events [36].

\section{Places and trajectories}

Place is the human way to understand and refer to space, and it goes well beyond geographic coordinates. Locations as simple coordinates are point-like, ubiquitous and precise. In contrast, places are not pointlike and have fuzzy boundaries determined by physical, cultural, and cognitive processes [106,81]. Furthermore, places, such downtown, can change their locations over time, just like physical objects [59]. In consequence, locations only insufficiently capture the identity and meaning of places.

So far, research in GIScience and geospatial semantics has been focusing on three major dimensions. Fist, the formalization of place [40], place data models [47], and place ontologies [1], in order to improve geographic information retrieval $[57,74,53]$. A promising direction of further research are affordance-based approaches towards place [58]. Second, the automated discovery of places, in order to enrich data with georeferences. A traditional direction of research is geoparsing, i.e., the discovery of places in texts by NLP methods, which can be also used to identify placerelated activities [5]. Recently, due to new technologies, research has focused on the discovery of places and user activities by mining (semantic) trajectories [112], which has also a tradition in ubiquitous computing [46]. Research also investigated how to reconstruct the spatial footprint of places based on geotags in social media, such as Flickr [49]. In the age of Big Data, semantic integration will allow researchers to combine data from heterogeneous sources to gain a more holistic understanding of places by studying locationbased social networks, different types of volunteered geographic information, authoritative data from the ground and via remote sensing, and many other data sources. Third, novel research addresses the design of place-based information systems [40] in which traditional operations and methods of GIS need to be redesigned to cope with places as referents. Geographic feature type ontologies are a central part of this vision.

\section{Sensor and observation semantics}

Naturally, observations play a key role in the geosciences, and, thus, also the involved sensors. In order to describe the origin and provenance of geodata, well-designed ontologies about sensors, observation, and measurement are necessary [67]. The so-called Semantic Sensor Web [99] develops ontologies, software, and methods to improve retrieval, access, and integration of observation data as well as sensor metadata. Ontologies, such as the Semantic Sensor Network ontology [23], provide formal specifications that ease retrieval and integration of data, while semanticsenabled Sensor Observation Services (SOS) provide access and querying capabilities [44]. Work on the Semantic Sensor Web also investigates how to establish and maintain provenance information about sensors, e.g., their survival range, sampling time, used observation procedure, and so forth $[113,85]$. To reduce manual interaction, sensor Plug \& Play investigates how to automatically register sensors and mediate their observation results to fit the needs of specific services [18]. Other examples of recent work include sensor data mashups [86] and research on stream reasoning [109]. An overview of research challenges for the Semantic Sensor Web was recently published by Corcho and Garcia-Castro [24].

\section{Similarity, alignment, and translation}

Semantic translation $[43,65,29,84]$, semantic similarity measurement $[92,90,71,98,83,53]$, and geoontology alignment [26] have been major research topics over the past years. Both are essential for establishing Semantic Reference Systems; while semantic translation maps between vocabularies and can be thought of as the analogy to datum transformation, semantic similarity measures the distance between concepts in a semantic space as an analogy to distance in space and time. Ontology alignment addresses the combination of multiple ontologies to enable data reuse and integration. The fact that most GI analysis, e.g. interpolation, kernel methods, or point pattern analysis, are based on spatial auto-correlation and distance in space, shows why semantic similarity is considered essential for making geo-ontologies and semantics first class citizens of GIS and spatial statistics. Similarity also plays a central role in most of the cognitive approaches introduced before, as these rely on direct mappings between ontologies instead of rigid top-down ontologies. However, as argued by Bittner et al. [13], these views do not contradict but can benefit from each other. Semantic similarity and analogy reasoning also enable novel types of user interfaces that ease navigating and browsing through geo-data and ontologies [53]. Similarity, however, is highly sensitive 
to context. Consequently, researchers have studied the impact of context and proposed different weights and procedures to account for its effect. A recent example for such work is Keßler's DIR measure, which identifies the contextual information with the largest impact on a given setting, and, thus, requiring adjustment of similarity measures [61].

\section{Spatial data infrastructures}

Spatial Data Infrastructures (SDI) provide standardized means for publishing, querying, retrieving, and accessing geodata via Web services. Additionally, SDIs offer notification and processing services and, thus, go beyond simple data stores. Data and processing services can be chained to model complex scientific workflows. To ensure a meaningful chaining, however, requires formal specifications of the service inputs, outputs, side effects, parameters, and so forth. Consequently, semantic markups for Web services have been an active research area for many years [79,31,105]. Examples of SDI specific research include the work of Lemmens et al. [70], Vaccari et al. [108], and Lutz [73].

While the Geo Web is typically composed of SDI services and uses its own markup languages and protocols, the Semantic Web is based on the its own technology stack. This leads to a situation were both infrastructures co-exist separately. It is, for instance, not possible to use a Semantic Web reasoner for instance classification of geo-data. Therefore, researchers developed different approaches for a semantic enablement of the Geo-Web. Janowicz et al., for instance, specified transparent and bi-directional proxies that allow users of both infrastructures to share data and services [54]. Semantic annotations have been proposed to lift existing geo-data to a semantic level $[62,80]$. In the context of the digital humanities, annotations have been used to create Linked Spatiotemporal Data and to enrich old maps with interlinked information from the global graph [101].

Finally, in the context of eScience and scientific workflows, researchers studied the role of semantic technologies and ontologies for the earth sciences [33, 16].

\section{Future challenges}

In the following, we highlight novel trends in geospatial semantics research that may set the agenda for future work.
Semantic engineering as interaction between humans

In recent work on data semantics [94,52], it has become apparent that semantic engineering of geographic information is often less a matter of informing about a static information ontology, i.e., an inventory of information concepts or realistic truth conditions, nor a matter of communication between machines, or of static mapping between ontologies. It can also be understood as a matter of dynamic machine mediated communication between humans [94], i.e., between data providers and users, with data (as well as semantic metadata) being the explicit top of a pyramid of implicit acts of interpretation, observation, and construction.

Data means something in a specific pragmatic context. However, this context is lost, in one way or another, under the conditions of digital communication. How can semantic engineering support the communication of data? The problem of sharing data via machines is not that machines are unable to communicate, but that humans misunderstand each other if communicating via machines [94]. Correspondingly, the task of semantic engineering is to support users in a communication situation, i.e., in reconstructing the meaning of data in a peer-to-peer fashion with respect to a provider context. The goal is to put humans in a position to compare their contexts, to judge fitness for purpose, and, thus, to generate semantic links in an ad-hoc fashion. This also implies that the common practice of publishing data together with static semantic annotations may rather hinder reusability and that dynamic typing approaches are required instead [52].

This requires a pragmatic shift in the role of existing semantic engineering technology: Semantic heterogeneity needs to be technologically supported instead of resolved. Semantic interoperability may be considered the outcome of a successful act of shared interpretations of certain classes. Users need to be put in a position where they can learn the provider classes or construct their own representations, if necessary. Information tools, such as formal specification, reasoning, as well as knowledge discovery tools, need to be reevaluated with respect to their role in this computer mediated communication process.

\section{Standardization and alignment}

As argued above, realizing the vision of a Geospatial Semantic Web will require combining top-down as well as bottom-up methods. Foundational ontologies, flexible ontology design patterns, and machine learn- 
ing should work hand in hand and have to be supported by a layered, provenance-enabled framework [51]. This has consequences for workflows in science and industry.

In the past, most work proposed to address the need for semantic interoperability by standardization. It was assumed that standardization bodies, such as the Open Geospatial Consortium, would provide the geosciences with a well defined set of ontologies that can be used to annotate data and services and, thus, ease retrieval and integration. There are some examples of success stories, e.g., GeoSPARQL, and a number of scientific communities were able to develop and maintain ontologies that are widely used, e.g., in bioinformatics. Nevertheless, sophisticated domain-level ontologies that go beyond simple taxonomies and have been adopted into scientific workflows are still rare. Developing such ontologies may take many years and revisions, raises questions of maintenance and of ontology evolution in light of new scientific discoveries, and may exclude the long tail of science by enforcing certain ontological commitments. It is also unclear how such ontologies would perform in interdisciplinary settings.

Alternatively, in the light of progress on ontology matching, aligning, and semantic translation [26,50, $100,35]$, local and purpose-driven micro-ontologies may be a suitable alternative. Such micro-ontologies have a limited scope, are potentially less affected by problems of uncertainty and vagueness, do not require users to agree to a large set of abstract ontological commitments, and are developed by communities or individuals interested in their maintenance. In fact, such ontologies are frequently used on the Linked Data Web. To ease retrieval and integration, such microontologies can be aligned and matched to other ontologies, including foundational ontologies. The resulting network of micro-ontologies can be used to reduce the risk of incompatibilities. It may turn out that the intuition that ontology standardization is less difficult and more persistent than investing into research on alignment, matching, and translation is wrong, and standardization is, in fact, the more difficult task. Consequently, one could standardize alignments instead of ontologies. This also shifts the focus from ensuring semantic interoperability to avoiding incompatibilities.

\section{Geospatial reasoning in the Semantic Web}

There are two traditions of geospatial reasoning: One is spatial reasoning, i.e., reasoning with qualita- tive spatial relations, including topological reasoning, such as overlap, meet and disjoint, and reasoning with directions ${ }^{1}$. This kind of reasoning is based on deductive inference in first-order predicate logic (FOL) [22], as well as on finite composition tables and constraint reasoning, in which all possible relations are enumerated exhaustively [91]. Another tradition of reasoning is based on spatial operators in a spatial database, i.e., on explicitly represented spatial geometry. These include point-in-polygon tests, R-tree search algorithms, and geometric as well as set-theoretic operators for vector data. Still another form is based on graph-based computational methods, which allow, e.g. to reason about road networks [20].

In comparison, current Semantic Web reasoning is rather narrowly defined. It focuses on particular decidable subsets of FOL, namely description logics and Horn rules, which lack the expressivity needed to reason with spatial relations [104]. Furthermore, other forms of geospatial reasoning, such as geometrical computation or approximate reasoning, are not well supported by the Semantic Web [48]. The integration of such reasoning paradigms into the Semantic Web requires further consideration of their RDF representation and computability, as well as a broadening of the existing reasoning paradigm itself. The latter may have been too narrowly focused on soundness, completeness, and decidability. It may, e.g., be useful to loosen soundness and completeness demands of proof procedures in order to allow for scalable approximate reasoning [48]. Furthermore, undecidable languages can be useful, since the decision problem is often sufficiently constrained in practice.

How can geospatial reasoning approaches best be integrated into the Semantic Web, in a way which allows tractable geospatial reasoning over Linked Spatiotemporal Data? Many spatial qualitative decision problems are NP-hard, however, tractable subsets can be identified [91]. There are attempts at integrating qualitative spatial reasoning into RDF reasoners, such as Racer [111] and Pellet [104]. A promising direction of research is to combine qualitative reasoners with geometrical computation. In the Semantic Web, this may be realized in terms of spatial extensions to RDF and SPARQL, such as stSPARQL or GeoSPARQL $[63,9]$.

\footnotetext{
${ }^{1}$ Prominent spatial calculi are mereotopological calculi, Frank's cardinal direction calculus, Freksa's double cross calculus, Egenhofer and Franzosa's 4- and 9-intersection calculi, Ligozat's flip-flop calculus, Cohn's region connection calculi (RCC), and the Oriented Point Relation Algebra [91].
} 


\section{Exploratory interaction and next-generation knowledge infrastructures}

The so-called Fourth Paradigm describes a new, data-intensive approach to scientific discovery [45]. It is often characterized as the scientific perspective on Big Data. The underlying argument is that the availability of data with a finer-grained spatial, temporal, and thematic resolution will allow scientists to answer complex questions which cannot be answered from within a single domain but span across multiple disciplines.

To address the arising new challenges and possibilities will require novel data management infrastructure to publish and retrieve scientific data and models across domains. NSF's future EarthCube is just one example for such a next-generation knowledge infrastructures. As community-driven initiative, EarthCube includes working groups focused on semantics and ontologies, brokering, as well as interoperability. Nevertheless, at their very core the envisioned next-generation infrastructures are mostly distributed and integrated knowledge archives. The crucial first step, namely data retrieval, is mostly neglected and approached following old paradigms or even (leaving simple taxonomies aside) semantics-free keyword search in metadata catalogs. Instead, next-generation knowledge infrastructures could be envisioned as distributed knowledge engines [52]. For example, by using deep semantics, reasoning services, and machine learning such knowledge engines could assist scientists in deriving and testing new theories and models. By applying analogy-based reasoning to Linked Spatiotemporal Data, such engines could also automatically propose relevant data sources for evaluation.

Finally, working with interdisciplinary data and models will require novel interaction paradigms and user interfaces that actively support scholars in finding relevant data. Instead of requiring precise queries and a detailed knowledge of the accessed data and cata$\log$, these new interfaces should support browsing and navigating the global graph of interlinked data. Combining semantic similarity and analogy reasoning with paradigms such as exploratory search [77], may be a promising direction for future research. To give a concrete example, scientists could query for the Paris of the West ${ }^{2}$, or the Californian Rattlesnake Fire of the 1990 s. Both queries would exploit the variety compo-

\footnotetext{
${ }^{2}$ and see how San Francisco would rank in the returned results.
}

nent of Big Data to take spatial, temporal, and thematic data from various sources, e.g., economics, human geography, climatology, geology, news records, and so forth into account [52].

\section{Conclusions}

How does the outlined past, present, and future research benefit the individual geoscientist? What is the value proposition of the Geospatial Semantic Web? Summing up, semantic technologies and ontologies support publishing, retrieving, and reusing data. They also reduce the risk that data is misinterpreted and, thus, improve the reproducibility of scientific results. The paradigm shift introduced by Linked (Open) Data breaks up data silos and allows to combine data onthe-fly. Creating smarter (meta-) data also supports the development of more generic and robust software. Scientists benefit from this by being able to select from a wide range of compatible (and free) Semantic Web software such as ontology editors, databases for RDF data, user interfaces, reasoners, as well as toolkits for triplification, linking, and annotation. Semantic technologies and ontologies also foster interoperability and data integration by supporting complex integrity constraint checking beyond topological consistency. Semantics-enabled user interfaces support scientist in exploring interdisciplinary datasets by browsing through interlinked data. Finally, semantic technologies can assist researchers by semi-automatically matching data to models and by translating between different conceptual schemas. Thus, they open up data sources and models that could not be used before.

\section{References}

[1] A.I. Abdelmoty, P. Smart and C.B. Jones, Building place ontologies for the semantic web: Issues and approaches, in: Proc. of the 4th ACM Workshop on Geographical Information Retrieval, GIR'07, ACM, New York, NY, USA, 2007, pp. 7-12.

[2] B. Adams and M. Raubal, A metric conceptual space algebra, in: Spatial Information Theory, 9th International Conference, COSIT 2009, Aber Wrac'h, France, September 21-25, 2009, Proc., K. Hornsby, C. Claramunt, M. Denis and G. Ligozat, eds, Springer, Berlin, 2009, pp. 51-68.

[3] P. Agouris and A. Stefanidis, Efficient summarization of spatiotemporal events, Commun. ACM 46(1) (2003), 65-66.

[4] O. Ahlqvist and A. Shortridge, Characterizing land cover structure with semantic variograms, in: Progress in Spatial Data Handling - 12th International Symposium on Spatial Data Handling, A. Riedl, W. Kainz and G. Elmes, eds, Springer, 2006, pp. 401-415. 
[5] A. Alazzawi, A. Abdelmoty and C. Jones, What can I do there? Towards the automatic discovery of place-related services and activities, International Journal of Geographical Information Science 26(2) (2012), 345-364.

[6] C. Alexander, S. Ishikawa and M. Silverstein, A Pattern Language: Towns, Buildings, Construction, Oxford University Press, New York, 1977.

[7] L. Barsalou, Perceptual symbol systems, Behavioral and Brain Sciences 22 (1999), 577-660.

[8] J. Bateman, Towards a generic foundation for spatial ontology, in: Proc. of the 3rd International Conference on For mal Ontology in Information Systems (FOIS-04), A. Varzi and L. Vieu, eds, IOS Press, Amsterdam, 2004, pp. 237-248.

[9] R. Battle and D. Kolas, Enabling the geospatial semantic web with parliament and GeoSPARQL, Semantic Web Journal 3(4) (2012).

[10] K. Beard, Modeling change in space and time: An event based approach, in: Dynamic and Mobile GIS: Investigating Changes in Space and Time, R. Billen, E. Joao and D. Forrest, eds, CRC Press, 2007, pp. 55-74.

[11] B. Bennett, Spatial vagueness, in: Methods for Handling Imperfect Spatial Information, R. Jeansoulin, O. Papini, H. Prade and S. Schockaert, eds, Springer-Verlag, 2011.

[12] B. Bennett, D. Mallenby and A. Third, An ontology for grounding vague geographic terms, in: Proc. 5th Intern. Conf. on Formal Ontology in Information Systems, C. Eschenbach and M. Gruninger, eds, IOS-Press [u.a.], Saarbrücken, 2008, pp. 280-293.

[13] T. Bittner, M. Donnelly and B. Smith, A spatio-temporal ontology for geographic information integration, International Journal of Geographical Information Science 23(6) (2009), 765-798.

[14] B. Brodaric, Geo-pragmatics for the geospatial semantic web, T. GIS 11(3) (2007), 453-477.

[15] B. Brodaric and M. Gahegan, Experiments to examine the situated nature of geoscientific concepts, Spatial Cognition \& Computation 7(1) (2007), 61-95.

[16] B. Brodaric and M. Gahegan, Ontology use for semantic escience, Semantic Web 1 (April 2010), 149-153.

[17] B. Brodaric and F. Probst, Dolce rocks: Integrating geoscience ontologies with dolce, in: AAAI Spring Symposium: Semantic Scientific Knowledge Integration, 2008, pp. 3-8.

[18] A. Bröring, P. Maué, K. Janowicz, D. Nüst and C. Malewski, Semantically-enabled sensor plug \& play for the sensor web, Sensors 11(8) (2011), 7568-7605.

[19] P. Burrough and A.U. Frank, ed., Geographic Objects with Indeterminate Boundaries, Taylor and Francis, 1996.

[20] N. Chrisman, Exploring Geographic Information Systems, Wiley, 2001.

[21] G. Christakos, P. Bogaert and M. Serre, Temporal GIS: Advanced Functions for Field-Based Applications, Springer Verlag, 2002.

[22] A.G. Cohn and S.M. Hazarika, Qualitative spatial representation and reasoning: An overview, Fundamenta Informaticae 46 (2001), 1-29.

[23] M. Compton, P. Barnaghi, L. Bermudez, R. Garcia-Castro, O. Corcho, S. Cox, J. Graybeal, M. Hauswirth, C. Henson, A. Herzog, V. Huang, K. Janowicz, W.D. Kelsey, D.L. Phuoc, L. Lefort, M. Leggieri, H. Neuhaus, A. Nikolov, K. Page, A. Passant, A. Sheth and K. Taylor, The ssn ontology of the w3c semantic sensor network incubator group, Web Seman- tics: Science, Services and Agents on the World Wide Web $\mathbf{0}(0)$ (2012).

[24] O. Corcho and R. Garcia-Castro, Five challenges for the semantic sensor web, Semantic Web 1(1-2) (2010), 121125.

[25] H. Couclelis, Ontologies of geographic information, International Journal of Geographical Information Science 24(12) (2010), 1785-1809.

[26] I. Cruz and W. Sunna, Structural alignment methods with applications to geospatial ontologies, Transactions in GIS, Special Issue on Semantic Similarity Measurement and Geospatial Applications 12(6) (2008), 683-711.

[27] A. Devaraju, Representing and reasoning about geographic occurrences in the sensor web, $\mathrm{PhD}$ thesis, University of Münster, 2012.

[28] M. Doerr, The cidoc conceptual reference module: An ontological approach to semantic interoperability of metadata, $A I$ Mag. 24(3) (Sept. 2003), 75-92.

[29] D. Dou, D.V. McDermott and P. Qi, Ontology translation on the semantic web, Journal of Data Semantics 2 (2005), 3557.

[30] M.J. Egenhofer, Toward the semantic geospatial web, in: Proc. of the 10th ACM International Symposium on Advances in Geographic Information Systems, GIS'02, ACM, 2002, pp. $1-4$.

[31] D. Fensel and C. Bussler, The web service modeling framework WSMF, Electronic Commerce Research and Applications 1(2) (2002), 113-137.

[32] F. Fonseca, M. Egenhofer, C. Davis and G. Câmara, Semantic granularity in ontology-driven geographic information systems, Annals of Mathematics and Artificial Intelligence 36(12) (2002), 121-151.

[33] P. Fox and J.A. Hendler, Semantic eScience: Encoding meaning in next-generation digitally enhanced science, in: The Fourth Paradigm: Data-Intensive Scientific Discovery, T. Hey, S. Tansley and K.M. Tolle, eds, Microsoft Research, 2009, pp. 147-152.

[34] A. Frank, Ontology for spatio-temporal databases, in: SpatioTemporal Databases: The Chorochronos Approach, Springer, Berlin, 2003, pp. 9-77.

[35] M. Gahegan, W. Smart, S. Masoud-Ansari and B. Whitehead, A semantic web map mediation service: Interactive redesign and sharing of map legends, in: Proc. of the 1st ACM SIGSPATIAL International Workshop on Spatial Semantics and Ontologies, SSO'11, ACM, 2011, pp. 1-8.

[36] A. Galton and R. Mizoguchi, The water falls but the waterfall does not fall: New perspectives on objects, processes and events, Applied Ontology 4(2) (2009), 71-107.

[37] A. Gangemi and V. Presutti, Towards a pattern science for the semantic web, Semantic Web 1(1-2) (2010), 61-68.

[38] P. Gärdenfors, Conceptual spaces as a framework for knowledge representation, Mind and Matter 2(2) (2004), 9-27.

[39] J. Gibson, The Ecological Approach to Visual Perception, Houghton Mifflin, Boston, 1979.

[40] M. Goodchild, Formalizing place in geographic information systems, in: Communities, Neighborhoods, and Health: Expanding the Boundaries of Place, Vol. 1, L.e.a. Burton, ed., Springer, Berlin, 2011, pp. 21-34.

[41] J. Goodwin, C. Dolbear and G. Hart, Geographical linked data: The administrative geography of great britain on the semantic web, Transactions in GIS 12 (2008), 19-30. 
[42] S. Harnad, The symbol grounding problem, Physica D 42 (1990), 335-346.

[43] F. Harvey, W. Kuhn, H. Pundt, Y. Bishr and C. Riedemann, Semantic interoperability: A central issue for sharing geographic information, The Annals of Regional Science 2(33) (1999), 213-232.

[44] C.A. Henson, J.K. Pschorr, A.P. Sheth and K. Thirunarayan, SemSOS: Semantic sensor observation service, in: International Symposium on Collaborative Technologies and Systems (CTS 2009), 2009.

[45] T. Hey, S. Tansley and K.M. Tolle, eds, The Fourth Paradigm: Data-Intensive Scientific Discovery, Microsoft Research, 2009.

[46] J. Hightower, S. Consolvo, A. LaMarca, I. Smith and J. Hughes, Learning and recognizing the places we go, in: UbiComp 2005: Ubiquitous Computing, M. Beigl, S. Intille, J. Rekimoto and H. Tokuda, eds, Lecture Notes in Computer Science, Vol. 3660, Springer, Berlin/Heidelberg, 2005 , pp. 903-903.

[47] L. Hill, Georeferencing. The Geographic Associations of Information, MIT Press, Cambridge, MA, 2006.

[48] P. Hitzler and F. van Harmelen, A reasonable semantic web, Semantic Web 1(1-2) (2010), 39-44.

[49] L. Hollenstein and R. Purves, Exploring place through usergenerated content: Using flickr tags to describe city cores, $J$. Spatial Information Science (2010), 21-48.

[50] P. Jain, P. Hitzler, A.P. Sheth, K. Verma and P.Z. Yeh, Ontology alignment for linked open data, in: The Semantic Web - ISWC 2010 - 9th International Semantic Web Conference, ISWC 2010, Shanghai, China, November 7-11, 2010, Revised Selected Papers, Part I, P.F. Patel-Schneider, Y. Pan, P. Hitzler, P. Mika, L. Zhang, J.Z. Pan, I. Horrocks and B. Glimm, eds, Lecture Notes in Computer Science, Vol. 6496, Springer, Heidelberg, 2010, pp. 402-417.

[51] K. Janowicz, Observation-driven geo-ontology engineering, Transactions in GIS, 2012, forthcoming.

[52] K. Janowicz and P. Hitzler, The digital earth as knowledge engine, Semantic Web Journal 3(3) (2012).

[53] K. Janowicz, M. Raubal and W. Kuhn, The semantics of similarity in geographic information retrieval, Journal of Spatial Information Science (2) (2011), 29-57.

[54] K. Janowicz, S. Schade, A. Bröring, C. Keßler, P. Maue and C. Stasch, Semantic enablement for spatial data infrastructures, Transactions in GIS 14(2) (2010), 111-129.

[55] M. Johnson, The Body in the Mind. The Bodily Basis of Meaning, Imagination and Reason, The University of Chicago Press, Chicago, 1987.

[56] C.B. Jones, A.I. Abdelmoty, D. Finch and G. Fu, The spirit spatial search engine: Architecture, ontologies and spatial indexing, in: Proc. 3rd Int. Conf. on Geographic Information Science, 2004, pp. 125-139.

[57] C.B. Jones, H. Alani and D. Tudhope, Geographical information retrieval with ontologies of place, in: Proc. of the International Conference on Spatial Information Theory: Foundations of Geographic Information Science, COSIT 2001, Springer-Verlag, London, UK, 2001, pp. 322-335.

[58] T. Jordan, M. Raubal, B. Gartrell and M.J. Egenhofer, An affordance-based model of place in GIS, in: 8th Int. Symposium on Spatial Data Handling (SDH'98), T. Poiker and N. Chrisman, eds, IUG, Vancouver, BC, 1998, pp. 98109.
[59] T. Kauppinen and E. Hyvönen, Modeling and Reasoning about Changes in Ontology Time Series, Springer-Verlag, New York, NY, 2007, pp. 319-338.

[60] M. Kavouras and M. Kokla, Theories of Geographic Concepts: Ontological Approaches to Semantic Integration, Taylor \& Francis, 2007.

[61] C. Keßler, What is the difference? A cognitive dissimilarity measure for information retrieval result sets, Knowl. Inf. Syst. 30(2) (Feb. 2012), 319-340.

[62] E. Klien, A rule-based strategy for the semantic annotation of geodatam, Transactions in GIS 11(3) (2007), 437-452.

[63] M. Koubarakis, K. Kyzirakos, M. Karpathiotakis, C. Nikolaou, M. Sioutis, S. Vassos, D. Michail, T. Herekakis, C. Kontoes and I. Papoutsis, Challenges for Qualitative Spatial Reasoning in Linked Geospatial Data, in: IJCAI 2011 Workshop on Benchmarks and Applications of Spatial Reasoning (BASR-11), 2011, pp. 33-38.

[64] W. Kuhn, Semantic reference systems (guest editorial), International Journal of Geographical Information Science 17(5) (2003), 405-409.

[65] W. Kuhn, Geospatial semantics: Why, of what and how? in: Journal on Data Semantics III, S. Spacciapietra and E. Zimányi, eds, Lecture notes in computer science, Vol. 3534, Springer, Berlin, 2005, pp. 1-24.

[66] W. Kuhn, An image-schematic account of spatial categories, in: Spatial Information Theory: 8th International Conference, COSIT 2007, Melbourne, Australia, September 19-23, 2007, Proc., Vol. 4736, S. Winter, M. Duckham, L. Kulik and B. Kuipers, eds, Springer, Berlin, 2007, pp. 152-168.

[67] W. Kuhn, A functional ontology of observation and measurement, in: GeoSpatial Semantics, K. Janowicz, M. Raubal and S. Levashkin, eds, LNCS, Vol. 5892, Springer-Verlag, Berlin, Heidelberg, 2009, pp. 26-43.

[68] W. Kuhn, Semantic engineering, in: Research Trends in Geographic Information Science, G. Navratil, ed., Lecture Notes in Geoinformation and Cartography, Vol. 12, Springer, Berlin, 2009, pp. 63-76.

[69] W. Kuhn, Modeling vs encoding for the semantic web, Semantic Web Journal 1(1-2) (2010).

[70] R. Lemmens, A. Wytzisk, R. de By, C. Granell, M. Gould and $\mathrm{P}$. van Oosterom, Integrating semantic and syntactic descriptions to chain geographic services, IEEE Internet Computing 10(5) (Sept. 2006), 42-52.

[71] B. Li and F. Fonseca, TDD - A comprehensive model for qualitative spatial similarity assessment, Spatial Cognition and Computation 6(1) (2006), 31-62.

[72] A. Llaves, H. Michels, P. Maue and M. Roth, Semantic event processing in envision, in: Proc. of the International Conference on Web Intelligence, Mining and Semantics (WIMS'12), 2012.

[73] M. Lutz, Ontology-based descriptions for semantic discovery and composition of geoprocessing services, GeoInformatica 11 (2007), 1-36.

[74] M. Lutz and E. Klien, Ontology-based retrieval of geographic information, International Journal of Geographical Information Science 20(3) (2006), 233-260.

[75] K. Lynch, The Image of the City, MIT Press, Cambridge, MA, 1960

[76] E. Mäkelä, E. Hyvönen and T. Ruotsalo, How to deal with massively heterogeneous cultural heritage data - lessons learned in culturesampo, Semantic Web 3(1) (2012), 85-109. 
[77] G. Marchionini, Exploratory search: From finding to understanding, Commun. ACM 49(4) (2006), 41-46.

[78] D.M. Mark, B. Smith, M. Egenhofer and S.C. Stephen Hirtle, Ontological foundations for geographic information science, in: Research Challenges in Geographic Information Science, R. McMaster and L. Usery, eds, CRC Press, 2004, pp. 335350 .

[79] D. Martin, M. Burstein, J. Hobbs, O. Lassila, D. Mcdermott, S. Mcilraith, S. Narayanan, M. Paolucci, B. Parsia, T. Payne, E. Sirin, N. Srinivasan and K. Sycara, Owl-s: Semantic markup for web services, November 2004.

[80] P. Maue, H. Michels and M. Roth, Injecting semantic annotations into (geospatial) web service descriptions, Semantic Web Journal 3(4) (2012).

[81] D. Montello, M. Goodchild, J. Gottsegen and P. Fohl, Where's downtown? Behavioral methods for determining referents of vague spatial queries, Spatial Cognition \& Computation 2(3) (2003), 185-204.

[82] NASA, A.40 computational modeling algorithms and cyberinfrastructure (December 19, 2011), Technical report, National Aeronautics and Space Administration (NASA), 2012.

[83] K. Nedas and M. Egenhofer, Spatial-scene similarity queries, Transactions in GIS 12(6) (2008), 661-681.

[84] N. Noy, Semantic integration: A survey of ontology-based approaches, SIGMOD Rec. 33 (December 2004), 65-70.

[85] P. Patni, S. Sahoo, C. Henson and A. Sheth Provenance aware linked sensor data, in: Proc. of the Second Workshop on Trust and Privacy on the Social and Semantic Web, P. Kärger, D. Olmedilla, A. Passant and A. Polleres, eds, 2010.

[86] D.L. Phuoc and M. Hauswirth, Linked open data in sensor data mashups, in: Proc. of the 2nd International Workshop on Semantic Sensor Networks (SSN09) in Conjunction with ISWC 2009, Vol. 522, D.D. Kerry Taylor, ed., CEUR, 2009.

[87] F. Probst, Observations, measurements and semantic reference spaces, Appl. Ontol. 3(1-2) (2008), 63-89.

[88] F. Probst and M. Lutz, Giving meaning to GI web service descriptions, in: International Workshop on Web Services: Modeling, Architecture and Infrastructure (WSMAI 2004), 2004.

[89] R. Raskin and M. Pan, Knowledge representation in the semantic web for earth and environmental terminology (sweet), Computers \& Geosciences 31(9) (2005), 1119-1125.

[90] M. Raubal, Formalizing conceptual spaces, in: Formal Ontology in Information Systems, Proc. of the Third International Conference (FOIS 2004), A. Varzi and L. Vieu, eds, Frontiers in Artificial Intelligence and Applications, Vol. 14, IOS Press, Torino, Italy, November 2004, pp. 153-164.

[91] J. Renz and B. Nebel, Qualitative spatial reasoning using constraint calculi, in: Handbook of Spatial Logics, M. Aiello, I. Pratt-Hartmann and J. van Benthem, eds, Springer-Verlag, 2007, pp. 161-215.

[92] A. Rodríguez and M. Egenhofer, Comparing geospatial entity classes: An asymmetric and context-dependent similarity measure, International Journal of Geographical Information Science 18(3) (2004), 229-256.

[93] S. Scheider, Grounding geographic Information in Perceptual Operations, Frontiers in Artificial Intelligence and its Applications, Vol. 244, IOS Press, 2012.

[94] S. Scheider, K. Janowicz and B. Adams, The observational roots of reference of the semantic web, CoRR, abs/1206.6347, 2012.
[95] S. Scheider, K. Janowicz and W. Kuhn, Grounding geographic categories in the meaningful environment, in: Spatial Information Theory, 9th International Conference, COSIT 2009, Aber Wrac'h, France, September 21-25, 2009, Proc., K. Hornsby, C. Claramunt, D.M. and G. Ligozat, eds, Springer, Berlin, 2009, pp. 69-87.

[96] S. Scheider and W. Kuhn, Affordance-based categorization of road network data using a grounded theory of channel networks, International Journal of Geographical Information Science 24(8) (2010), 1249-1267.

[97] C. Schlieder, Digital heritage: Semantic challenges of long-term preservation, Semantic Web 1(1-2) (2010), 143 147.

[98] A. Schwering and M. Raubal, Measuring semantic similarity between geospatial conceptual regions, in: GeoSpatial Semantics, First International Conference, GeoS 2005, Mexico City, Mexico, November 29-30, 2005, Proc., M.A. Rodríguez, I.F. Cruz, M.J. Egenhofer and S. Levashkin, eds, Lecture Notes in Computer Science, Vol. 3799, Springer, 2005, pp. 90-106.

[99] A. Sheth, C. Henson and S. Sahoo, Semantic sensor web, IEEE Internet Computing (2008), 78-83.

[100] P. Shvaiko and J. Euzenat, Ten challenges for ontology matching, in: On the Move to Meaningful Internet Systems: OTM 2008, R. Meersman and Z. Tari, eds, LNCS, Vol. 5332, Springer, 2008, pp. 1164-1182.

[101] R. Simon, C. Sadilek, J. Korb, M. Baldauf and B. Haslhofer, Tag clouds and old maps: Annotations as linked spatiotemporal data in the cultural heritage domain, in: Workshop on Linked Spatiotemporal Data 2010, Held in Conjunction with the 6th International Conference on Geographic Information Science (GIScience 2010), Vol. 691, CEUR-WS, Zurich, Switzerland, 2010.

[102] B. Smith and D.M. Mark, Ontology and geographic kinds, in: Proc., International Symposium on Spatial Data Handling, Vancouver, Canada, 12-15 July, 1998.

[103] C. Stadler, J. Lehmann, K. Höffner and S. Auer, Linkedgeodata: A core for a web of spatial open data, Semantic Web Journal 3(4) (2012).

[104] M. Stocker and E. Sirin, PelletSpatial: A hybrid RCC-8 and RDF/OWL reasoning and query engine, in: Proc. of the 5th International Workshop on OWL: Experiences and Directions (OWLED), 2009.

[105] P. Szekely, C.A. Knoblock, S. Gupta, M. Taheriyan and B. Wu, Exploiting semantics of web services for geospatial data fusion, in: Proc. of the 1st ACM SIGSPATIAL International Workshop on Spatial Semantics and Ontologies, SSO'11, ACM, 2011, pp. 32-39.

[106] Y. Tuan, Space and Place: Humanistic Perspective, Theory and Decision Library, D. Reidel Pub. Co., 1979.

[107] E.L. Usery and D. Varanka, Design and development of linked data from the national map, Semantic Web Journal 3(4) (2012).

[108] L. Vaccari, P. Shvaiko and M. Marchese, A geo-service semantic integration in spatial data infrastructures, International Journal of Spatial Data Infrastructures Research 4 (2009).

[109] E.D. Valle, S. Ceri, F.v. Harmelen and D. Fensel, It's a streaming world! Reasoning upon rapidly changing information. IEEE Intelligent Systems 24(6) (Nov. 2009), 8389. 
[110] W. van Hage, V. Malaise, R. Segers, L. Hollink and G. Schreiber, Design and use of the simple event model (SEM), Web Semantics: Science, Services and Agents on the World Wide Web 9(2) (2011).

[111] M. Wessel and R. Möller, Flexible software architectures for ontology-based information systems, Journal of Applied Logic 7(1) (2009), 75-99

[112] J.J.-C. Ying, W.-C. Lee, T.-C. Weng and V.S. Tseng, Semantic trajectory mining for location prediction, in: 19th ACM SIGSPATIAL International Symposium on Advances in Geographic Information Systems, ACM-GIS 2011, Proc., I.F.
Cruz, D. Agrawal, C.S. Jensen, E. Ofek and E. Tanin, eds, ACM, 2011, pp. 34-43.

[113] S. Zednik, P. Fox, D.L. McGuinness, P.P. da Silva and C. Chang, Semantic provenance for science data products: Application to image data processing, in: Proc. of the First International Workshop on the Role of Semantic Web in Provenance Management (SWPM 2009), Collocated with the 8th International Semantic Web Conference (ISWC-2009), CEUR Workshop Proc., Vol. 526, CEUR-WS.org, 2009. 